\title{
Density limit in helicon discharges
}

\section{David G Miljak $\dagger$ and Francis F Chen}

University of California, Los Angeles, CA 90095-1594, USA

Received 3 April 1998, in final form 28 July 1998

\begin{abstract}
To produce the highest possible density in a helicon discharge, two helical antennas were used to launch $m=+1$ waves colliding at the midplane. With $2 \mathrm{~kW}$ of RF power on each antenna, a maximum plasma density of $8 \times 10^{13} \mathrm{~cm}^{-3}$ was achieved, not much higher than with a single antenna. This density limit is explained by gas depletion, and a method to avoid this is suggested.
\end{abstract}

\section{Introduction}

The suitability of helicon discharges for producing high density plasmas for etching semiconductor chips has been known for over a decade [1]. Peak densities of order $10^{14} \mathrm{~cm}^{-3}$ have been reported in plasmas with only axial magnetic confinement $[2,3]$. If another half to one order of magnitude can be achieved, such plasmas would be useful for accelerator applications. Plasma accelerators [4] in which electrons surf on maximum-amplitude plasma waves have an acceleration gradient up to $\sim 1 \sqrt{ } n \mathrm{eV} \mathrm{cm}^{-1}$, where the electron density $n$ is in $\mathrm{cm}^{-3}$. Thus, $1 \mathrm{GeV} \mathrm{cm}^{-1}$ is the limit if $n=10^{18} \mathrm{~cm}^{-3}$, or realistically, $\leq 100 \mathrm{MeV} \mathrm{cm}^{-1}$ if only a fraction of the wavebreaking limit can be attained. Still, this is much higher than possible conventionally. The plasma must be uniform to about $\pm 1 \%$ both radially and axially; these conditions have been given by Chen [5]. Beat-wave accelerators operate in this regime, but so far plasmas only a few $\mathrm{mm}$ long are possible at such densities. Wakefield accelerators [4], on the other hand, can operate at lower densities, requiring $1 \mathrm{~m}$ of $10^{15} \mathrm{~cm}^{-3}$ plasma for $300 \mathrm{MeV}$ at $10 \%$ of wavebreaking. Plasma lens concepts [4] also require such plasmas. Conceptual schemes for staged accelerators with a series of plasma sources have been given by Chen $[5,6]$. The purpose of this work is to test the density limit in RF plasma sources.

The $m=+1$ (right-hand circularly polarized) mode of the helicon dispersion relation has been found in numerous laboratories [7-11] to produce much higher densities than the $m=-1$ or linearly polarized modes. We therefore employ right-hand helical antennas to excite this mode preferentially. We have also used other density-enhancing tricks, described below, though their effectiveness is not completely understood. At these high densities, probe tips have to be made of carbon, and the discharge has to be pulsed to preserve the probes. The radial and axial scales of helicon discharges in long cylinders make it much easier to satisfy uniformity requirements than with laser ionization. However, we shall find that straightforward scaling of $10^{13} \mathrm{~cm}^{-3}$ discharges by increasing the RF power

$\dagger$ Present address: Research School of Physical Sciences and Engineering, Australian National University, Canberra, ACT 0200, Australia.

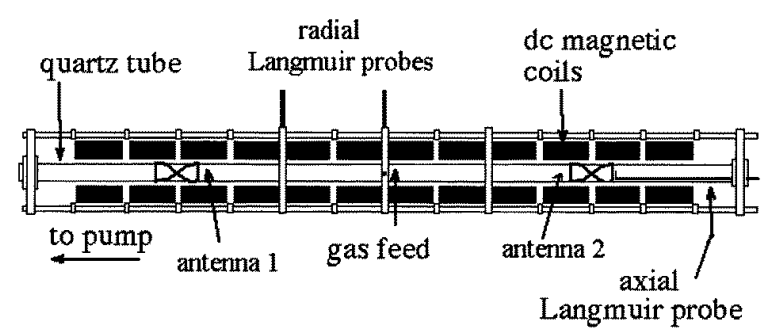

Figure 1. Diagram of the apparatus.

cannot extend the density beyond about $10^{14} \mathrm{~cm}^{-3}$. This ceiling, we believe, is due to gas depletion; if true, higher densities can be obtained by proper design of the vacuum system.

\section{Apparatus}

Experiments were performed in a $4.5 \mathrm{~cm}$ diameter quartz tube $1.65 \mathrm{~m}$ long, as shown in figure 1, pumped at one end. Argon gas could be fed either at the pump end or near the midplane (normal position) without affecting the results. For later experiments at $1200 \mathrm{G}$, a carbon block was positioned at the throat of the discharge tube at the pump end, obstructing about $80 \%$ of the cross section. Previous experiments [12] have shown that this acts to reduce the ion-pump effect, increasing the neutral gas supply to the discharge. Coils of radius $1.2 \mathrm{~cm}$ larger than the quartz tube produced an axial magnetic field $\boldsymbol{B}_{0}$ of up to $1200 \mathrm{G}$. The discharge pulse duration was $50 \mathrm{~ms}$, with a duty cycle of about $1 \%$.

Radiofrequency (RF) power was delivered via either one or two half-wavelength, helical antennas, each of the proper helicity to launch $m=+1$ waves toward the midplane. Both antennas were $10 \mathrm{~cm}$ long, an optimal length found in previous tests $[3,13]$. As shown in figure 1, a right-helical antenna was positioned at the pump end, and a left-helical antenna at the opposite end, with $\boldsymbol{B}_{0}$ in the $+z$ direction (away from the pump). RF power was provided by two $2 \mathrm{~kW}, 27.12 \mathrm{MHz}$ amplifiers, each with its own 


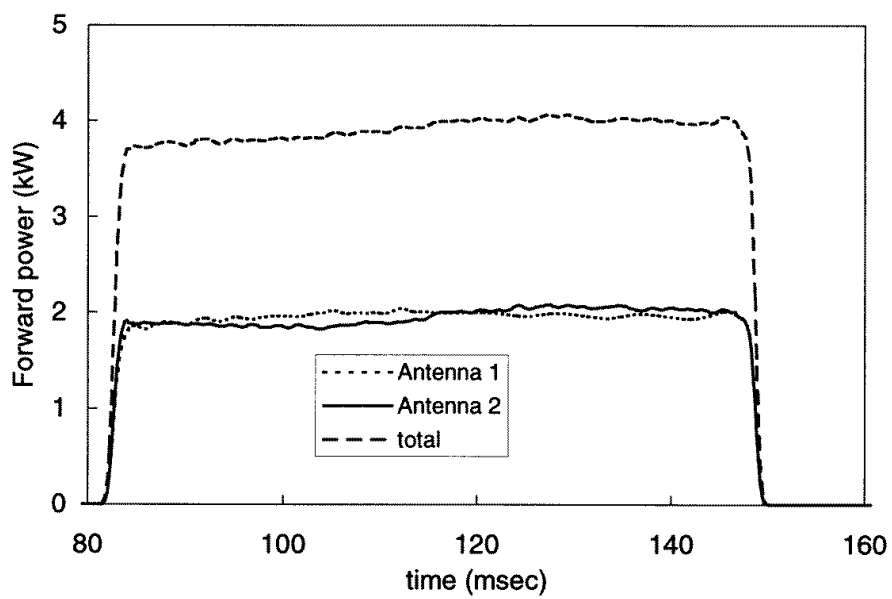

Figure 2. Time evolution of forward power to each antenna.

matching circuit. The supplies could be fed separately to each antenna or combined efficiently to couple $4 \mathrm{~kW}$ into a single antenna. Forward and reflected power were monitored on each antenna, and the circuit was retuned for each measurement to maintain $<0.5 \%$ reflection. Figure 2 shows the forward powers in a typical pulse. A current transformer was also used to monitor the RF current in the RH antenna. These RF diagnostics allowed the calculation of resistive antenna loading, as detailed in previous work [13]. Antenna loading was found to be insensitive to the parameter range scanned in this experiment, staying between 1.8 and $2.0 \Omega$. This was the case despite doubling the power from 2 to $4 \mathrm{~kW}$ and varying the pressure between 10 and 160 mTorr. Given a vacuum loading of $0.35 \Omega$, the coupling efficiency (defined as the ratio of power input to the plasma to the available power from the amplifier) was about $80 \%$ for all results to be presented.

Axial density $n_{e}$, temperature $T_{e}$ and floating potential $V_{f}$ profiles at $r=0$ were built up on a shot-to-shot basis using a fully RF-compensated axial dogleg Langmuir probe of previously published design [14]. The probe voltage was linearly swept over a $5 \mathrm{~ms}$ period. Analysis of the $I-V$ characteristic was restricted to the region around the floating potential. At these high densities, the ion saturation current could be assumed to be flat, giving least squares errors of less than $0.5 \mathrm{eV}$. However, for positions under the antennas, distortion of the probe characteristic by the intense RF fields there increased the errors to $\pm 1 \mathrm{eV}$.

\section{Results}

\subsection{Single-antenna results}

To establish a basis for comparison, measurements were first made with a single antenna at the pump end of the machine. Axial density profiles at $800 \mathrm{G}$ and 20 mTorr are shown in figure 3 for 2 and $4 \mathrm{~kW}$ of RF power. For these results, there was no carbon block in the device, and the end-coil currents were set for uniform magnetic field. The twofold increase in power gave only a modest increase in maximum density, with almost no change in the vicinity of the antenna. The ion saturation currents in figure 3(a) show a more marked difference, but this appears to be due to the temperature increase at $4 \mathrm{~kW}$, shown in figure 3(b). This saturation of the density at $5 \times 10^{13} \mathrm{~cm}^{-3}$ is consistent with the value of $8 \times 10^{13} \mathrm{~cm}^{-3}$ observed previously [3] at $1200 \mathrm{G}$ and higher pressures. The result can be understood in terms of the well known helicon dispersion in its simplest form:

$$
\omega=\left(k_{\perp}^{2}+k_{\|}^{2}\right)^{1 / 2} \frac{k_{\|} B}{n e \mu_{0}}
$$

where $k_{\perp}$ and $k_{\|}$are the perpendicular and parallel wavenumbers, $B$ the magnetic field and $n$ the electron density. If one assumes that $k_{\|}$is set by the length of the antenna and $k_{\perp}$ by the tube radius, then a given value of $B$ determines $n$, regardless of RF power. An increase of power, then, can be accommodated only by an increase in $T_{e}$ or a broadening of the density profile. Though $n$ was normally measured only at $r=0$, a few radial sweeps made by rotating the dogleg probe showed little deviation from the density profile, measured at $2 \mathrm{~kW}$, shown in figure 4. Thus, the saturation in density is consistent, to lowest order, with equation (1), but further experiments with dual antennas contradict such a simple explanation.

\subsection{Dual-antenna results at $800 \mathrm{G}$}

With two antennas, one at each end of the machine, it was anticipated that higher peak densities could be achieved by overlapping the downstream high-density regions produced by each antenna. Figure 5 compares the performance of a twin-antenna system with that of a single antenna. The $4 \mathrm{~kW}$, single-antenna result of figure 3 is reproduced, together with the case of two antennas, each at $2 \mathrm{~kW}$, separated by $95 \mathrm{~cm}$. This is the maximum possible separation for magnetic fields uniform through the antennas. The loading of each antenna was only weakly affected by the existence of the other antenna, and only slight retuning of the matching circuits was necessary. The dual-antenna density profiles exhibit higher peak densities, as well as a higher axially integrated density. For the single antenna the temperature peaks $20 \mathrm{~cm}$ from the antenna centre, while the 

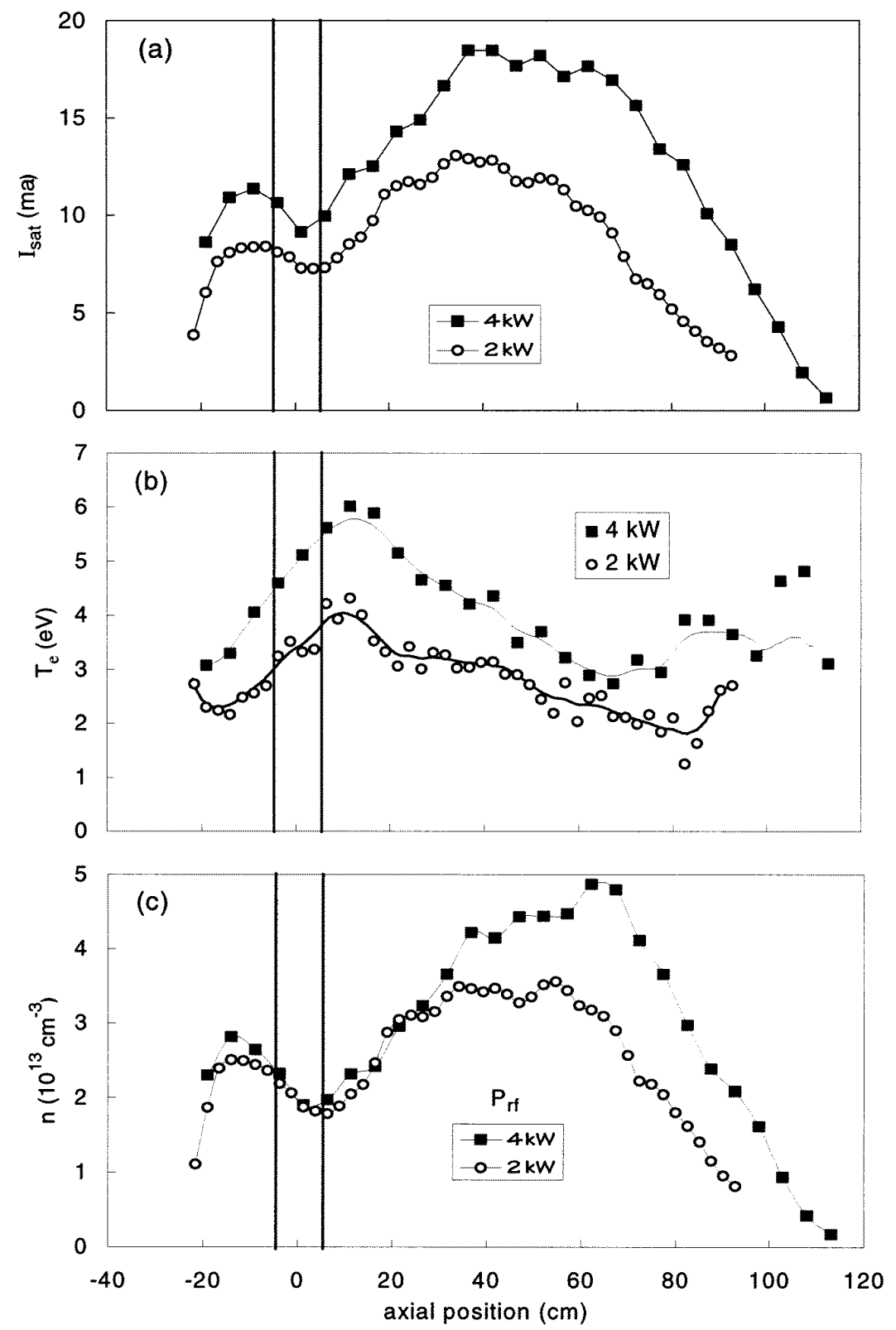

Figure 3. Comparison of plasma parameters for single antenna excitation at $800 \mathrm{G}$ (uniform field) and $20 \mathrm{mTorr}$, for RF powers of 2 and $4 \mathrm{~kW}$ : (a) ion saturation current; (b) electron temperature; (c) plasma density. The ends of the antenna are indicated by the vertical bars.

density peaks $60 \mathrm{~cm}$ from the centre. For the two-antenna case, the temperature peaks have similar axial offsets from the antennas, but the density maxima are closer to the respective antennas. Rather than overlapping to double the density, the density maxima move apart to maintain a relative minimum at the midplane.

Previous results [10] have shown that the axial density variation can largely be explained by electron pressure balance along $B$; that is, as $K T_{e}$ decays away from the antenna, $n$ increases so as to keep $n K T_{e}$ approximately constant. To check this, we measured the axial variation of the floating potential $V_{f}$ and obtained, with the $T_{e}$ data, the space potential $V_{s}$ for the single- and dual-antenna cases (figure 6). The parallel forces $-e n E$ and $-\nabla p$ computed from this, shown in figure 7 , indeed vary in opposite directions, but their magnitudes do not agree. The discrepancy is more pronounced for the dual-antenna case. Other mechanisms must be at play, as evidenced also by the faster decay of $n$ between its peak and the midplane when a second antenna is added. These effects will be discussed in section 4.

\subsection{Dual antenna results at $1200 \mathrm{G}$}

At this point we made a number of changes in an attempt to increase the density above $10^{14} \mathrm{~cm}^{-3}$ : (a) $B$ was increased by $50 \%$ to $1200 \mathrm{G}$, (b) the antenna spacing was reduced by $20 \mathrm{~cm}$ to $75 \mathrm{~cm}$; (c) the magnetic field was flared 


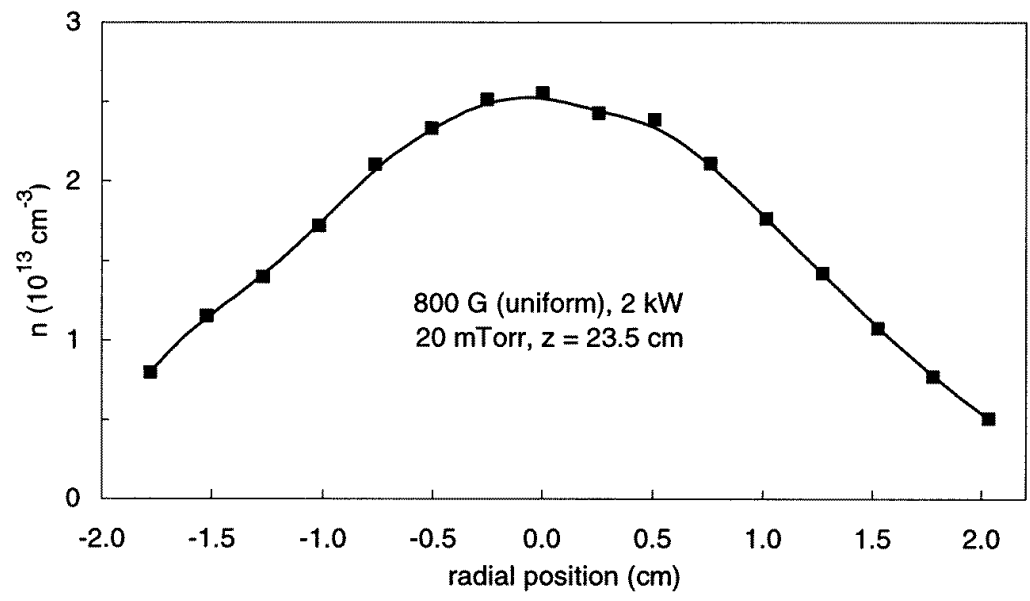

Figure 4. Radial electron density profile at $z=23.5 \mathrm{~cm}$ for single antenna excitation, with the same parameters as in figure 3; $P_{r f}=2 \mathrm{~kW}, B=800 \mathrm{G}, p=20$ mTorr.

in the antenna region; (d) a carbon block was inserted into the pump end of the tube behind the antenna and (e) higher pressures were scanned to find an optimum. Previous experiments have shown that cusp fields and obstacles behind the antenna could increase the peak density by about a factor of two [12], but the effect was not entirely explained. In the present experiment the cusp field decreased the density under the antenna but did not have any effect on the downstream density.

Under these conditions, the discharge reached equilibrium only after a transient state. Over most of the pressure range between 10 and 160 mTorr, a sharp transition was observed early in the discharge. Before the transition, a transient state (up to $10 \mathrm{~ms}$ long) was characterized by very high axial nonuniformity, with high ion saturation current observed close to the antennas but dropping significantly between the antennas. After the transition, a stable plasma was observed with higher axial uniformity. The time evolution of the ion saturation current at three axial locations is shown in figure 8 for a pressure of 63 mTorr, at which the transition can be seen clearly. Axial profiles of plasma parameters corresponding to the transient and stable phases are shown in figure 9. For the transient phase the electron temperatures were difficult to obtain accurately, owing to variations in ion saturation current over the duration of a sweep. Nevertheless, the axial nonuniformity for this case is obviously very severe. The peak level of ion saturation in the pre-transition phase of the discharge could be varied by changing the pressure. Lower pressures caused the transient phase to have a shorter duration, but with a higher peak level. At low enough pressure $(<20$ mTorr $)$ the transient phase was very short or non-existent, with the discharge settling quickly to its final equilibrium, as it did at $800 \mathrm{G}$ without the carbon block. At lower pressure still ( $<5$ mTorr), kilohertz instabilities in the plasma column were very strong and precluded any serious measurements. At very high pressures (>120 mTorr) the transition was smeared into a gradual evolution, and a final equilibrium state could be reached only after lengthening the pulse.

The transition observed appears to be a change in discharge equilibrium from a state characterized by gas depletion and large axial gradients to a relaxed state with small axial gradients and forces, resulting in a filling-in of the hollow region between the antennas. The duration of the transient state is consistent with that for a redistribution of the neutral density. Helicon plasmas have been found to be efficient ion pumps; that is, ionized neutrals do not flow to the pump at their normal thermal speed but are accelerated by the pre-sheath potential to the ion acoustic velocity, which is an order of magnitude higher. Consequently, the presence of plasma of density $>10^{13} \mathrm{~cm}^{-3}$ greatly reduces the neutral density below its initial fill value. The ion pumping effect was at first inferred indirectly [10] but has since been measured [15]; it will be evaluated in section 4 .

Compared to the results at $800 \mathrm{G}$, the decrease in antenna separation for this set of data had the general effect of increasing plasma uniformity, at least for the post-transition phase of the discharge. At low pressure, after the transition, one broad density peak was produced between the two antennas. By increasing the pressure, the density associated with this broad peak was increased. Past a certain critical pressure however, the single broad peak split into two separate peaks. This critical pressure was found to be 63 mTorr, corresponding to the results shown in figure 9. Increasing the pressure to 160 mTorr results in the data of figure 10, where the axial nonuniformity is clearly evident. At this pressure, doubling the power with a second antenna increases not the peak density, but only the temperature.

Thus, the post-transition plasma of figure 9 gives the highest possible density with axial uniformity. The axial density variation is approximately $10 \%$ for the range $z=$ $20-70 \mathrm{~cm}$. This figure takes into account shot-to-shot reproducibility as well as any real density gradients that may exist. The density of $8 \times 10^{13} \mathrm{~cm}^{-3}$ obtained in figure 9 , although very high by normal plasma processing standards, may be too low by a factor of 5 or 10 for accelerator applications. It was not possible to increase the density by raising the pressure and moving the antennas closer together in order to overlap the density maxima from each antenna. For pressures above the critical pressure 

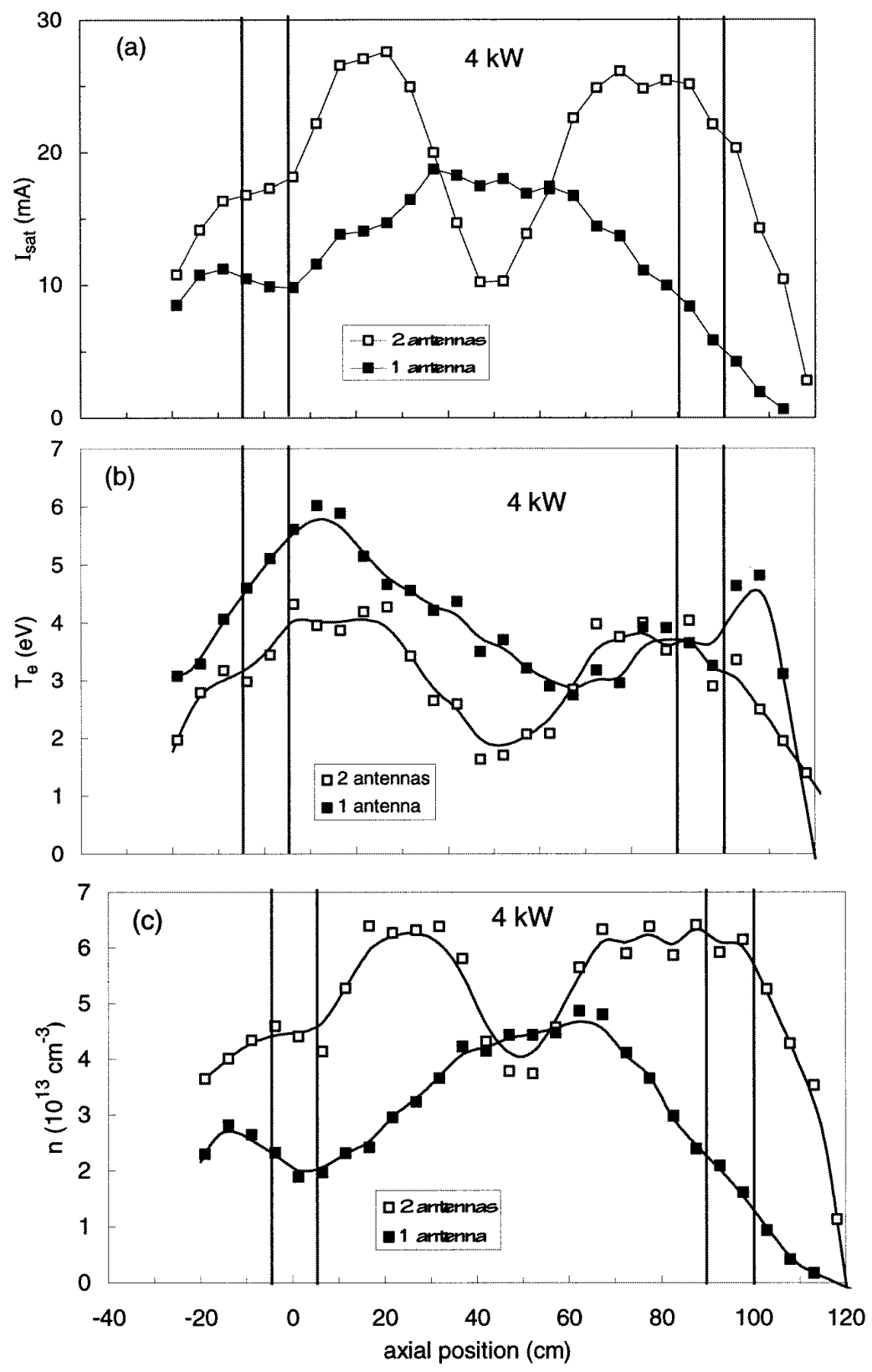

Figure 5. Comparison of plasma parameters for single- and dual-antenna excitation at $800 \mathrm{G}$ (uniform field), $20 \mathrm{mTorr}$ and $4 \mathrm{~kW}$ (total): (a) ion saturation current; (b) electron temperature; (c) plasma density.

of 63 mTorr, a density minimum, rather than maximum, always appeared at the midplane in the stable phase.

The density minimum suggests that some sort of destructive interference could be occurring with dual antennas. Axial beat patterns of helicon waves have indeed been seen in the past with a single antenna [16]. This explanation, however, fails on three counts: (1) the beat patterns from each antenna cannot interfere destructively, since they are fluctuations in $|B|$ without regard to phase; (2) the wavelengths of the waves are much shorter than the scalelength of the density dip and (3) downstream ionization has only a minor effect on the density in any case [10]. As a further check, the experiment was repeated [17] with antennas powered from oscillators that were not phase locked to each other, as they were for the above data. Figure 11 shows the ion saturation current at $1200 \mathrm{G}$ and 160 mTorr with two antennas driven by independent $1.4 \mathrm{~kW}$ power supplies at 27.12 and $13.56 \mathrm{MHz}$. A deep minimum is seen at the midplane; the minimum in density would be shallower, but no correction for $T_{e}$ variations was possible in this case. We believe that the minimum can only be caused by gas depletion.

\section{Analysis}

The effect of the ion pumping mechanism can be estimated as follows. First, we compute the gas inlet rate $q_{0}$ per mTorr of fill pressure $p_{0}$ from the known pumping speed $S$ 

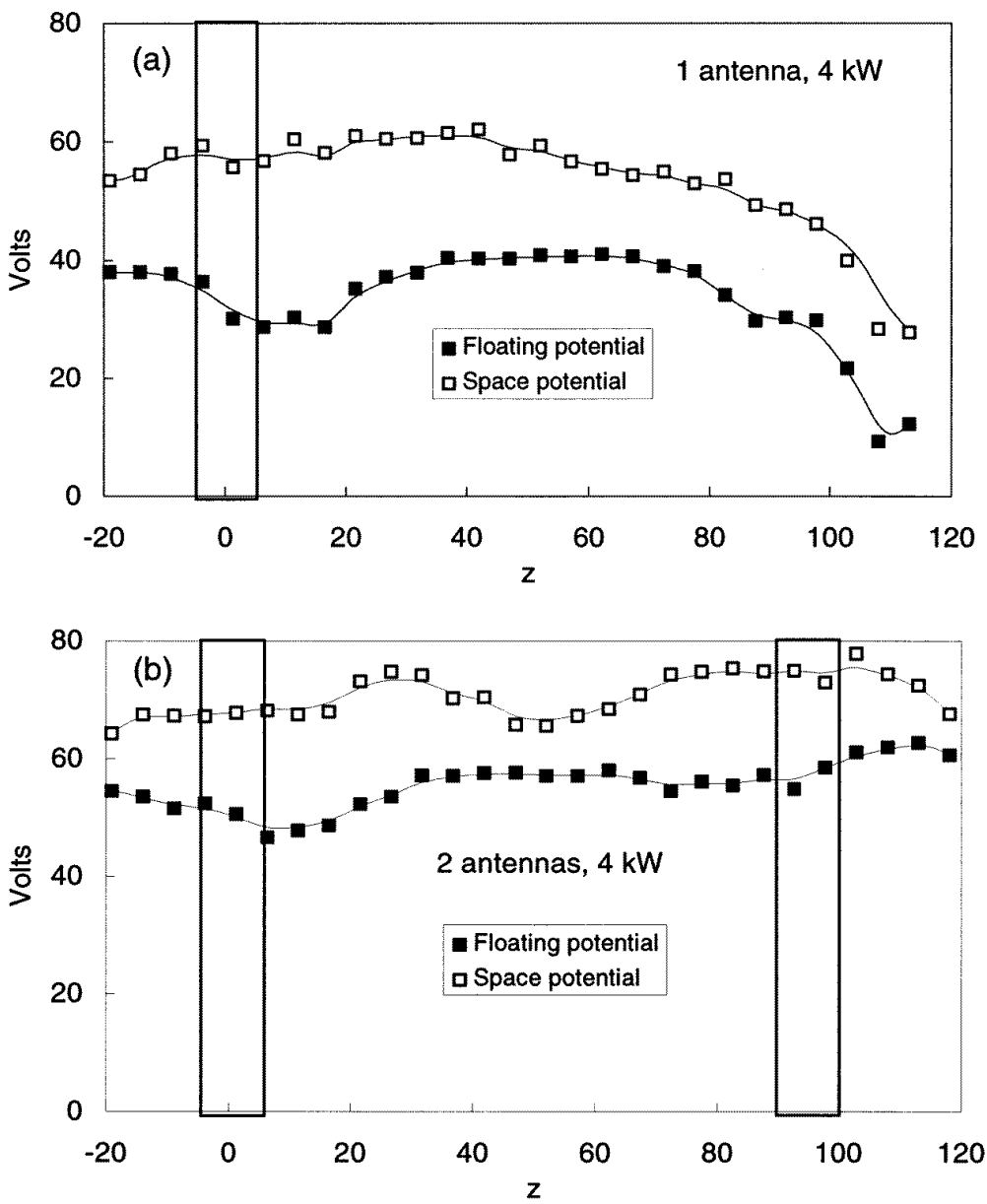

Figure 6. Axial profiles for the floating potential and the space potential (computed from the measured temperature). Operating conditions are as in figure 5.

of the pump. Thus, before the discharge is turned on, the neutral density $n_{n 0}$ is given by

$$
q_{0} p_{0}=S n_{n 0}\left(p_{0}\right)
$$

After a plasma of average density $\langle n\rangle$ is created over a cross sectional area $A$, the flow of ions to the pump (where they recombine and are pumped out) is given by $\langle n\rangle A c_{s}$, where $c_{s}$ is the ion sound speed $c_{s}=\left(K T_{e} / M\right)^{1 / 2}$. The remaining neutrals of density $n_{n}$ are pumped out at the rate $S$. Since the input rate is unchanged, the neutral density in the presence of plasma is given by

$$
n_{n}=\left(q_{0} p_{0}-\langle n\rangle A c_{s}\right) / S \text {. }
$$

We see that $n_{n}$ is reduced to zero if the plasma density is too high. Figure 12(a) shows $n_{n}$ as a function of $\langle n\rangle$ for various fill pressures $p_{0}$.

The equilibrium plasma density $n$ is reached when the ionization rate equals the loss rate. The ionization rate $Q$ is simply $\left\langle n_{e}\right\rangle n_{n}\langle\sigma v\rangle_{i o n}$. Though the ionization probability $\langle\sigma v\rangle_{i o n}$ is a function of $T_{e}$, for this estimate it is sufficient to take $K T_{e}=3 \mathrm{eV}$, as we did in computing $c_{s}$. The function $Q(n)$ is shown in figure 12(b). To estimate the loss rate in a simple fashion, we must make some physical assumptions.
The electrons are assumed to follow the Boltzmann relation

$$
n_{e}=n_{0} \exp \left(e \phi / K T_{e}\right)
$$

even in the presence of a magnetic field. Electrons are known to communicate across field lines in devious ways in order to thermalize more rapidly than classical theory would predict (Langmuir's paradox). Because of this unpredictability, we do not attempt to compute the transverse diffusion rate of the electrons. Rather, we assume that the radial losses are controlled by the loss rate of the nearly unmagnetized ions, and that the electrons will follow the ions in order to preserve quasineutrality. In steady state, the radial component of the ion equation of motion is

$$
e n E_{r}-\gamma_{i} K T_{i} n^{\prime}-M n v_{i 0} v_{r}=0
$$

where $n^{\prime}=\partial n / \partial r$, and $E_{r}$ is given by equation (4):

$$
E_{r}=-\frac{K T_{e}}{e} \frac{n^{\prime}}{n} .
$$

Neglecting the $T_{i}$ contribution to $c_{s}$, we obtain from equations (5) and (6)

$$
v_{r}=-\frac{c_{s}^{2}}{v_{i 0}} \frac{n^{\prime}}{n} .
$$



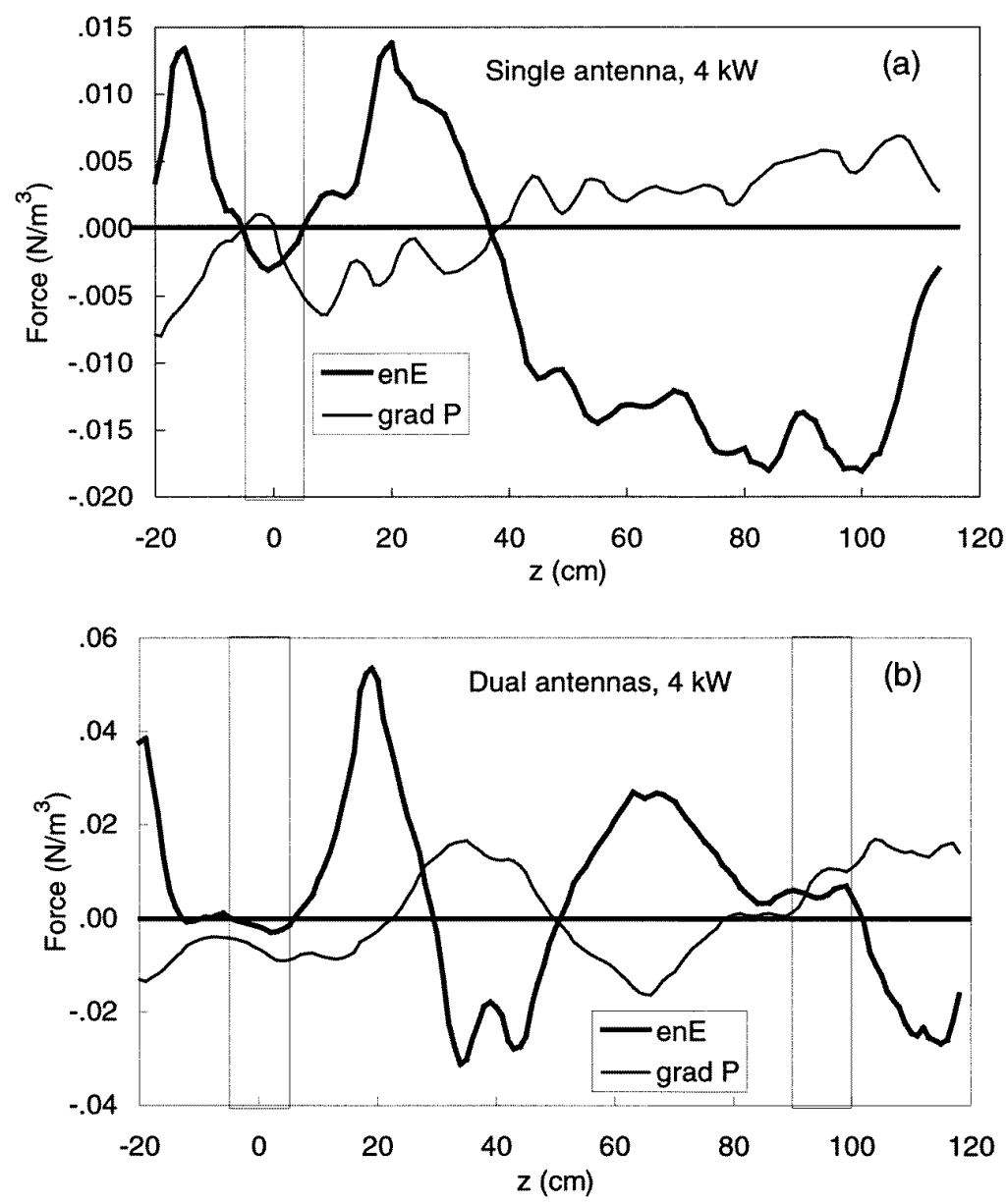

Figure 7. Values of the two dominant terms in the axial force balance equation calculated from the data of figures 5 and 6 , for the cases of single- and dual-antenna excitation. The opposite signs of the electric and pressure gradient forces shows a tendency toward electron pressure balance along $\boldsymbol{B}$.

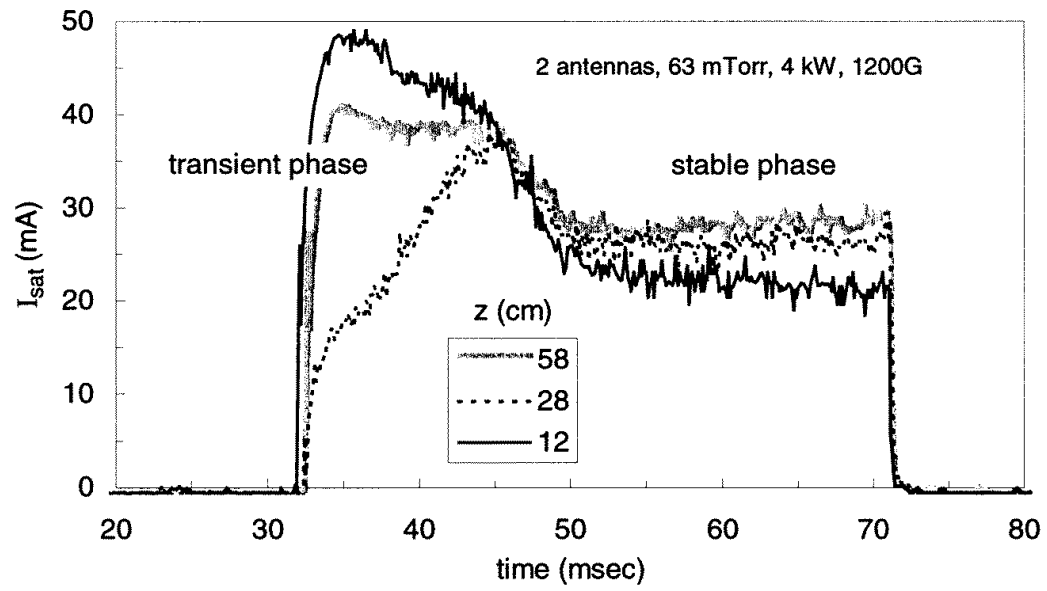

Figure 8. Temporal evolution of the ion saturation current at $1200 \mathrm{G}$ and $63 \mathrm{mTorr}$ at three axial positions $z$ : $12 \mathrm{~cm}$ (close to antenna 1), $28 \mathrm{~cm}$ (close to the midplane) and $58 \mathrm{~cm}$ (close to antenna 2).

The ion-neutral collision frequency is given by $v_{i 0}=$ $e / M \mu$, where $\mu=1.2 \times 10^{6} / p_{0} \mathrm{~cm}^{2} \mathrm{~s}^{-1} \mathrm{~V}^{-1}$ is the measured mobility of $\mathrm{Ar}^{+}$in $\mathrm{Ar}$ [18]. The plasma loss rate is given by the integral of $n v_{r}$ over the cylindrical surface at $r=a$. We may neglect the axial losses, which also scale as $K T_{e}$ but with a much longer scalelength. To evaluate equation (7), we approximate the profile of figure 4 with the parabola

$$
n=n_{0}\left(1-\frac{r^{2}}{w^{2}}\right)
$$



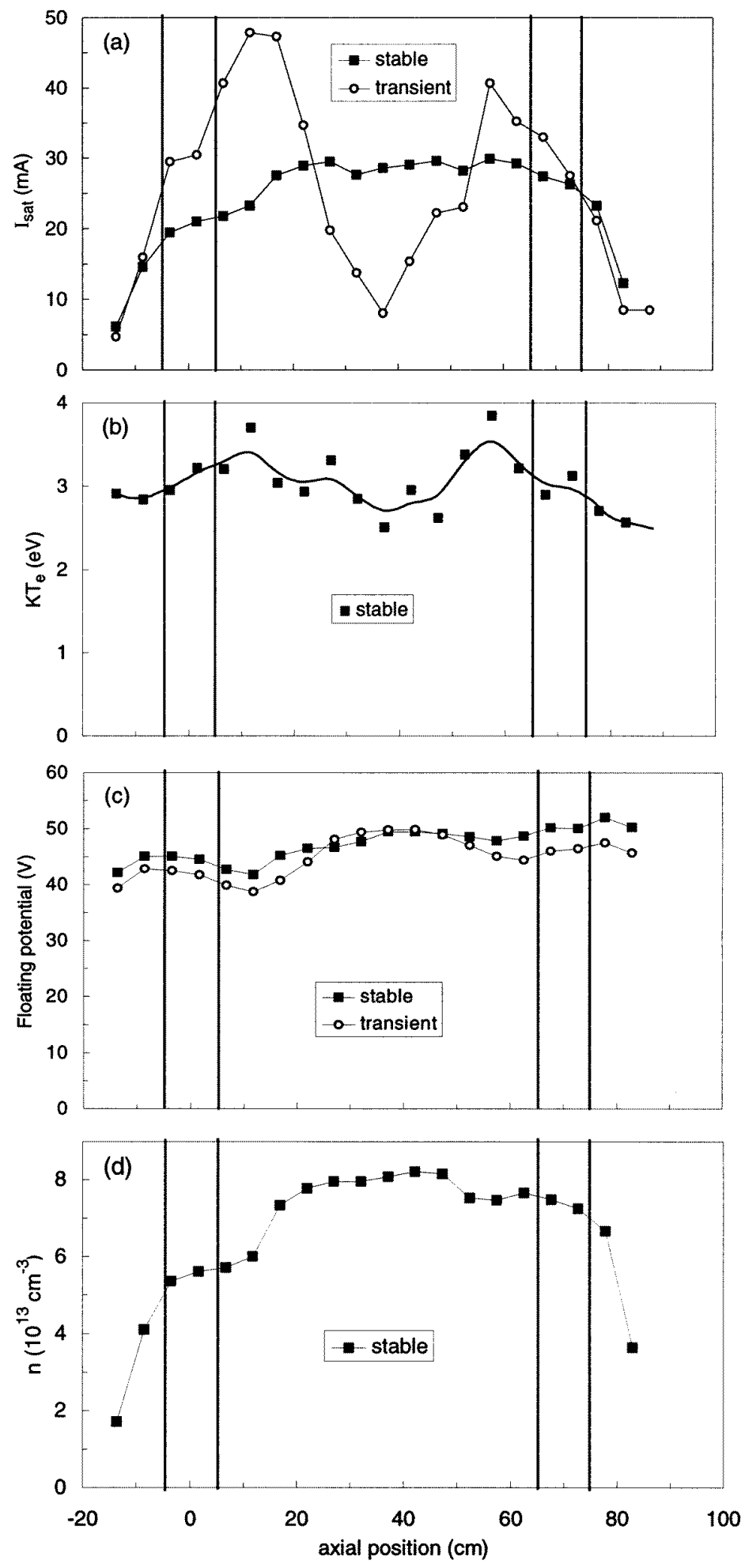

Figure 9. Axial profiles of (a) ion saturation current and (c) floating potential for the transient and stable phases of the discharge at $1200 \mathrm{G}$ and $63 \mathrm{mTorr}$, taken at $t=36$ and $65 \mathrm{~ms}$, respectively (figure 8). Axial profiles of electron temperature and density in the stable phase are shown in (b) and (d).

with $a^{2} / w^{2}=0.8$; this yields a value $\langle n\rangle=0.6 n_{0}$ for use in equation (3). Since equation (7) diverges as $v_{i 0} \rightarrow 0$, in principle a flux limiter should be used when the mean free path becomes too long at low neutral densities.
However, for our parameters this point is barely reached at equilibrium. The computed loss rates are shown in figure 12 (b) and intersect the ionization rate curves at $\langle n\rangle \approx$ $4 \times 10^{13} \mathrm{~cm}^{-3}$ for 160 mTorr and $\approx 1.5 \times 10^{13} \mathrm{~cm}^{-3}$ for 

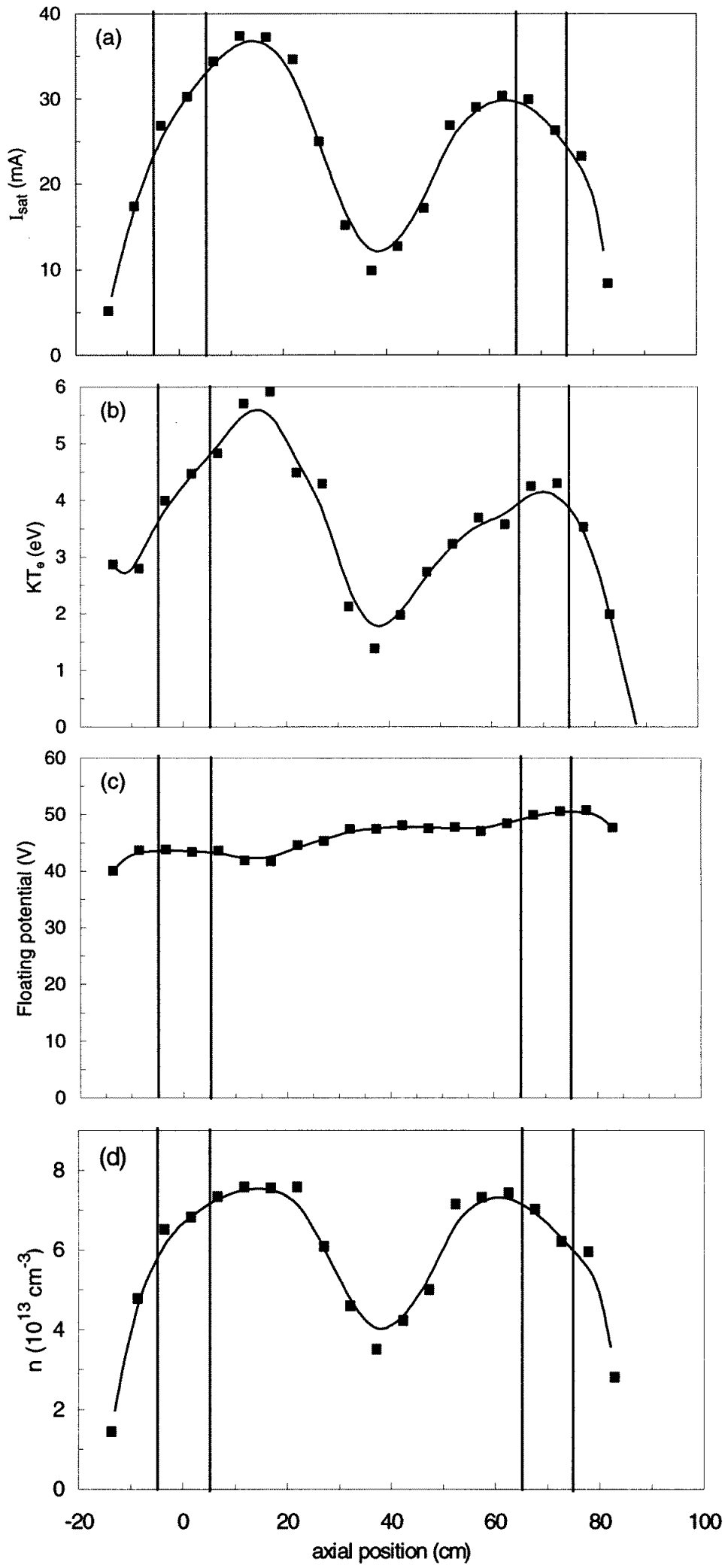

Figure 10. Axial profiles of (a) ion saturation current, (b) electron temperature, (c) floating potential and (d) plasma density at $1200 \mathrm{G}$ and 160 mTorr. This pressure is above the critical pressure for axial uniformity.

63 mTorr, in excellent agreement with the observed density limits considering the simplicity of this zero-dimensional calculation.
Apparently what happens in high-intensity, highpressure, dual-antenna helicon discharges is the complete depletion of neutrals at the midplane. The ionized neutrals 


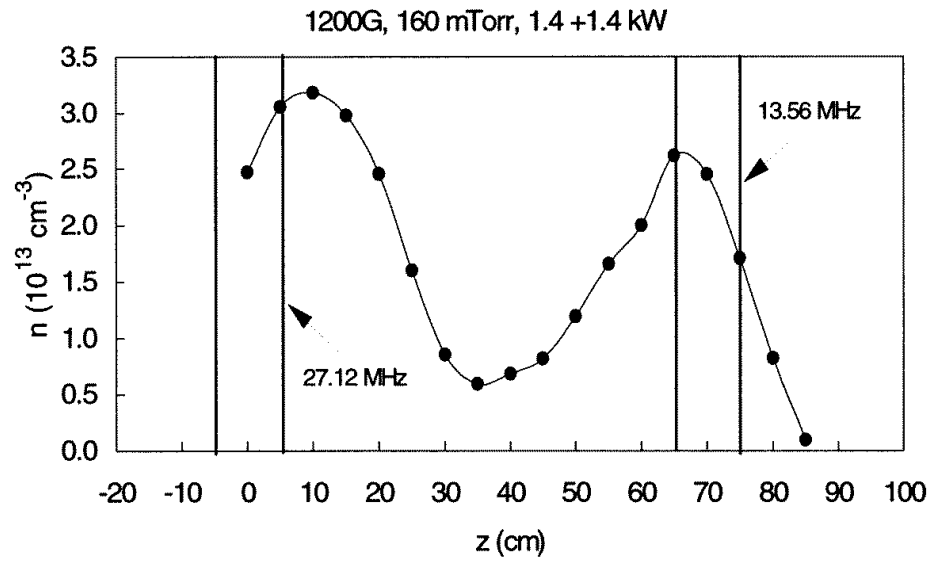

Figure 11. Axial density profile at $1200 \mathrm{G}$ and $160 \mathrm{mTorr}$, with dual antennas driven at $1.4 \mathrm{~kW}$ each at different frequencies [17].
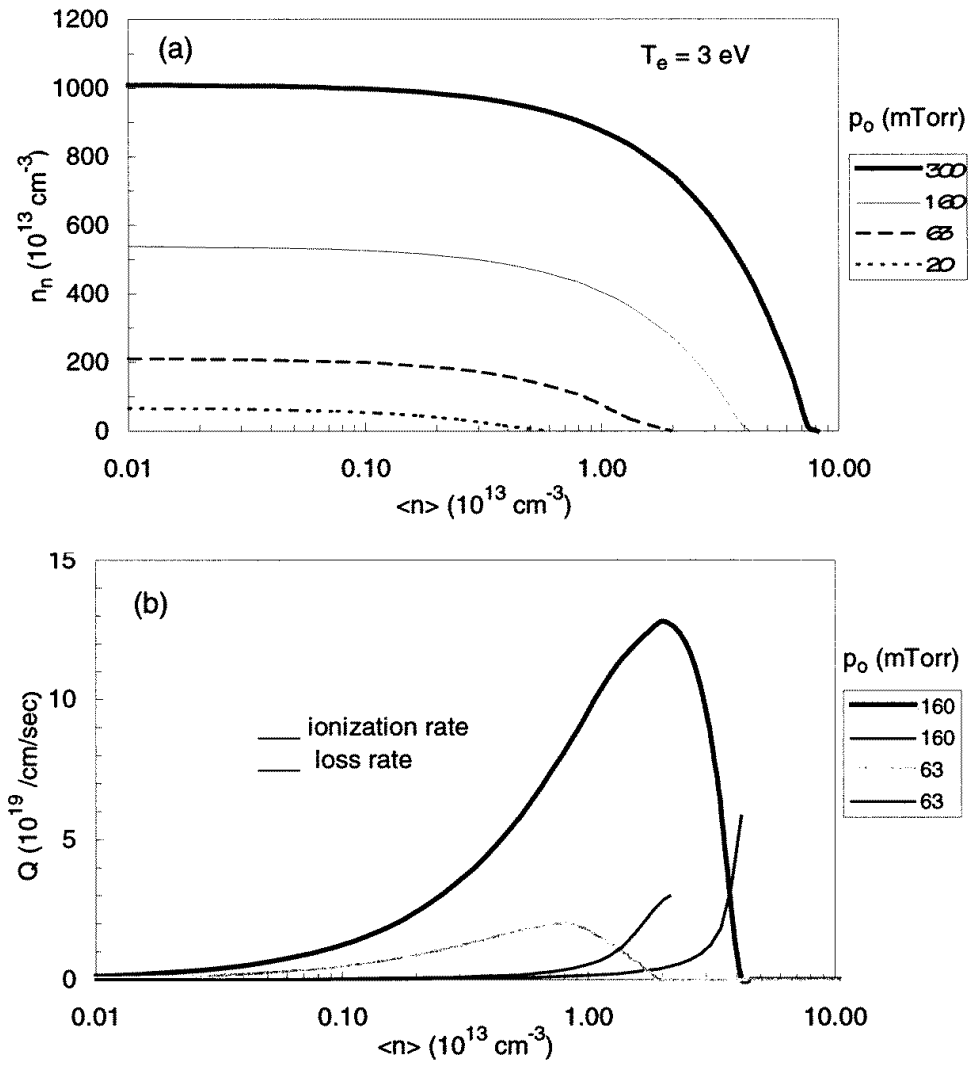

Figure 12. Computations of ion pumping effect: (a) neutral density against average plasma density at different filling pressures; (b) ionization rate and ion loss rate per unit length against average plasma density at two pressures.

are driven at the acoustic velocity to the endplates (one of which is the carbon block), where they recombine and flow back into the discharge. However, these refluxing neutrals cannot reach the midplane because their mean free path against ionization by a $10^{14} \mathrm{~cm}^{-3}$ plasma is only $\approx 2 \mathrm{~cm}$. Thus a high neutral density builds up in the antenna region, increasing the rate of ionization there by coupled helicon/Trivelpiece-Gould waves [19]. Plasma produced in this highly collisional region is lost radially before it can reach the midplane. Downstream ionization does not occur because of the absence of neutrals and because the damping length of H/TG waves is likely to be short in the highdensity region. This picture would explain why the density peaks occur closer to the antenna at higher pressures and why a density minimum can occur at the midplane.

\section{Conclusions and projections}

By applying $2 \mathrm{~kW}$ of RF power to each of two helical antennas separated by $75 \mathrm{~cm}$, a plasma of $8 \times 10^{13} \mathrm{~cm}^{-3}$ 
density, uniform to $\pm 5 \%$ over $50 \mathrm{~cm}$, has been generated in 63 mTorr of argon with a $1.2 \mathrm{kG}$ magnetic field. Attempts to increase the density by increasing the pressure failed because a density minimum appeared at the midplane, and the separated density maxima did not exceed the density achievable with a single antenna. This behaviour was explained by the depletion of neutral gas between the antennas due to the ion pumping effect at these high densities. These results suggest that higher densities can be achieved with better gas feed. For instance, gas puffing can be used, or a large plenum [6] can be built around the plasma column so that stored gas can be fed radially into the plasma during the pulse. With axially uniform neutral fuelling, the high degree of ionization in these devices assures that no large axial gradients in either potential or density can occur, and axial scalelengths of the order of metres should be routinely achievable. Since the radii of such discharges are of the order of centimetres, the radial scalelengths should be much larger than those obtained with laser ionization.

The possibility of exceeding the density limit of $\approx 10^{14} \mathrm{~cm}^{-3}$, however, leads to surprising conclusions. Consider a large cylindrical chamber (figure 13) of radius $R(\approx 20 \mathrm{~cm})$ and length $L(\approx 1 \mathrm{~m})$ with helicon sources of radius $a(\approx 1 \mathrm{~cm})$ at each end injecting $m=+1$ waves toward the midplane. The chamber is filled initially with $p_{0}$ mTorr of argon at $25^{\circ} \mathrm{C}$, and the stored gas flows radially into the plasma of radius $a \approx 1 \mathrm{~cm}$ when the discharge is pulsed on. Ionized neutrals are ion-pumped through the small tubes into large pumping stations, where the recombined neutrals are removed; to be conservative, we assume no refluxing of the gas. The acoustic transit time across $R$ is of order $0.6 \mathrm{~ms}$, so that transient density gradients will have decayed a few milliseconds after the plasma is fired, and the gas will flow inward in steady, shear-free molecular flow with a mean free path of $\approx 1 \mathrm{~cm}$ at 5 mTorr. As a neutral atom enters the plasma, its mean free path $\lambda_{m}$ against ionization varies from 18 to $0.2 \mathrm{~cm}$ as $T_{e}$ varies from 2 to $4 \mathrm{eV}$, for $n_{e} \approx 10^{14} \mathrm{~cm}^{-3}$. Consider first the simple case of $T_{e}<3 \mathrm{eV}$, when $\lambda_{m}$ is not $\ll a$ and $n_{n}$ can be considered to be distributed throughout the plasma.

The flow of neutrals into the plasma at $r=a$ is

$$
\Gamma_{i n}=2 \pi a L n_{n} \bar{v} / 4
$$

where $\bar{v} \approx 4 \times 10^{4} \mathrm{~cm} \mathrm{~s}^{-1}$ is the argon thermal velocity at $300 \mathrm{~K}$. The rate at which neutrals are consumed is

$$
\Gamma_{\text {loss }}=f \pi a^{2} \operatorname{Ln} n_{n} n_{e} P_{i}
$$

where $P_{i}=\langle\sigma v\rangle_{\text {ion }}$ is the ionization probability and $f$ is a form factor accounting for the mild radial gradient in $n_{n}$. Equating (9) and (10), we find that $n_{n}$ cannot be found this way, while $n_{e}$ is given by

$$
n_{e}=\bar{v} / 2 a P_{i} f
$$

regardless of $p_{0}$. For $a=1 \mathrm{~cm}$, and $P_{i}=2 \times 10^{-10} \mathrm{~cm}^{3} \mathrm{~s}^{-1}$ (argon at $K T_{e}=3 \mathrm{eV}$ ), and $f \approx 1$, the density is fixed at $n_{e} \times 10^{14} \mathrm{~cm}^{-3}$. This density apparently suffices to ionize completely any number of neutrals that may be injected. The plasma radius $a$ cannot be reduced further because of diffusion, and $\bar{v}$ can be increased only by using a lighter gas or by heating it. It would be counterproductive to increase the RF power, since that would lead to higher $T_{e}$, and thus higher $P_{i}$. To increase $n_{e}$ beyond $10^{14} \mathrm{~cm}^{-3}$, one would have to use a gas with a lower value of $P_{i}$ and allow $T_{e}$ to decay away from the antennas to decrease $P_{i}$ further.

The calculation above can be made more realistic by accounting for refluxing of the neutrals. For $1 \mathrm{~m}$ long discharges, the dominant ion loss is in the radial direction, but this does not represent a loss of neutrals, since the escaping ions flow to the end walls of the gas plenum and recombine there to replenish $n_{n}$. The ions escaping along $B_{0}$, however, recombine in a pumping chamber, where the neutrals are removed. Assuming that the ion density at the sheath edge is about $n_{i} / 2$, we can write the ion loss rate as

$$
\Gamma_{i z}=2 \pi a^{2} \frac{1}{2} n_{i} c_{s}
$$

where $c_{s}$ is the ion acoustic velocity. Equating this to $\Gamma_{i n}$ in equation (9), we obtain

$$
n_{n}=\frac{2 a c_{s}}{L \bar{v}} n_{i} \cong 0.14 n_{i}
$$

for our illustrative parameters. Thus a pressure of less than $1 \mathrm{mT}$ Torr is required at a density of $10^{14} \mathrm{~cm}^{-3}$, as far as neutral balance is concerned. Ion balance, however, is more stringent. The number of ions created per second is equal to the rate of ionization given in equation (10). The loss of ions by radial diffusion against neutrals is

$$
\Gamma_{r}=2 \pi a L n v_{r} \quad n v_{r}=-D_{i 0} \mathrm{~d} n / \mathrm{d} r \approx D_{i 0} n / \frac{1}{2} a
$$

where $D_{i 0}=T_{i}(\mathrm{eV}) \mu$. Setting equations (10) and (14) equal to each other yields

$$
p_{0}^{2}=1.4 \times 10^{-7} T_{i} / f a^{2} P_{i}
$$

For $T_{i}=0.1 \mathrm{eV}, a=1 \mathrm{~cm}, P_{i}=2 \times 10^{-10} \mathrm{~cm}^{3} \mathrm{~s}^{-1}$ $\left(T_{e}=3 \mathrm{eV}\right)$ and $f=0.7$, the required pressure is $p_{0} \approx 10$ mTorr, regardless of $n$.

At densities well above $10^{14} \mathrm{~cm}^{-3}$, the neutrals will be ionized in a skin layer before reaching the axis, and the plasma created near the surface will diffuse both inward and outward from there. Ions diffusing outward will flow to the end walls of the plenum and recombine there; as before, this flow does not represent a loss of neutrals. Ions diffusing inward will form a squarish, slightly hollow density profile and will be lost by axial flow into the pumping sections. The neutral density $n_{n}$ for $r<a$ now falls inward from the outside value $n_{a} \equiv n_{n}(a)$, as described by the continuity equation

$$
\frac{1}{r} \frac{\partial}{\partial r}\left(r n_{n} v_{r}\right)=-n_{n} n_{e} P_{i}
$$

Here, $v_{r}$ is the fluid velocity, which we assume to be constant and approximately equal to $-\bar{v} / 4$. The solution of equation (16) is

$$
r n_{n}=a n_{a} \mathrm{e}^{-(a-r) / \delta_{i}}
$$




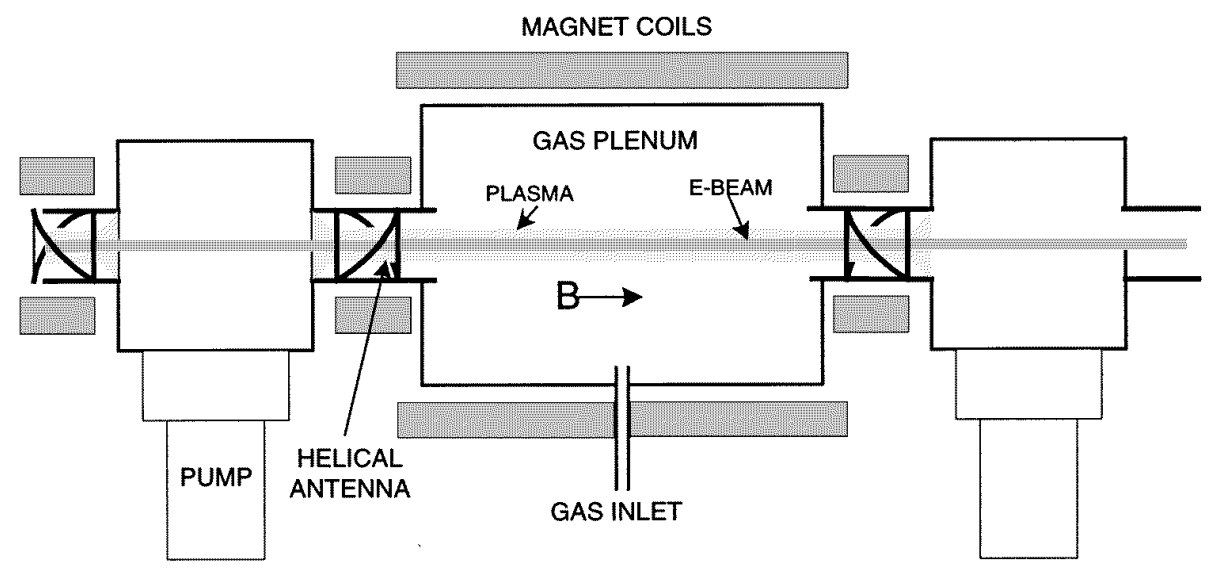

Figure 13. Conceptual sketch of an ultrahigh-density helicon source with a gas plenum.

where

$$
\delta_{i}=\frac{\bar{v}}{4 n_{e} P_{i}}
$$

is the ionization skin depth. The total ionization rate is given by

$$
\frac{\mathrm{d} N_{i}}{\mathrm{~d} t}=L \int_{0}^{a} n_{n} n_{e} P_{i} 2 \pi r \mathrm{~d} r=2 \pi a L n_{a} n_{e} P_{i} \delta_{i}\left(1-\mathrm{e}^{-a / \delta_{i}}\right) .
$$

Here we have used equation (17) and assumed uniform $n_{e}$. Equating this to the axial loss rate given by equation (12), we can solve for the required neutral density $n_{a}$ :

$$
n_{a}=\frac{a c_{s}}{2 L P_{i} \delta_{i}}=2 \frac{a}{L} \frac{c_{s}}{\bar{v}} n_{e}=\left(8 \pi \frac{T_{e}}{T_{0}}\right)^{1 / 2} \frac{a}{L} n_{e}
$$

where $T_{0}$ is the neutral temperature. Note that the use of equation (18) has caused the sensitive function $P_{i}\left(T_{e}\right)$ to cancel out. For $a=1 \mathrm{~cm}, L=1 \mathrm{~m}$ and $T_{e}=3 \mathrm{eV}$, we find that a pressure of 10 mTorr will provide neutral balance up to $2 \times 10^{15} \mathrm{~cm}^{-3}$. For ion balance, we can use equations (19) and (18) for the ion creation rate and equation (14) for the ion loss rate, except that the density scalelength $a / 2$ should be replaced by $\delta_{i}$ or something of that order. These equations then give

$$
p_{0}^{2}=3.6 \times 10^{-16} T_{i} P_{i} n^{2}
$$

which has the opposite $T_{e}$ dependence and an $n$ dependence compared with the lower density case of equation (15). For $T_{i}=0.1 \mathrm{eV}$ and $T_{e}=3 \mathrm{eV}$, we then have for the required pressure

$$
p_{0}=8.5 n_{14} \text { mTorr }
$$

where $n_{14}$ is plasma density in units of $10^{14} \mathrm{~cm}^{-3}$.

The plenum radius $R$ affects only the pressure decay time $t_{p}$. Since $N_{n}=\pi R^{2} L n_{n}$ and $\Gamma_{i n}$ is given by equation (9), we have

$$
t_{p} \approx \frac{N_{n}}{\Gamma_{i n}}=\frac{2 R^{2}}{a \bar{v}}
$$

yielding a time of $20 \mathrm{~ms}$ for $R=20 \mathrm{~cm}$. Thus, a pulse length of $5 \mathrm{~ms}$ or so would be long enough for the pressure to come into equilibrium and yet short enough that $n_{n}$ does not change appreciably during the pulse. Alternatively, one could design the plenum to have a radial acoustic resonance in the $\mathrm{kHz}$ regime, so that the plasma can be pulsed in synchronism with pressure maxima at $r=a$.

Finally, we consider the RF power $P_{r f}$ needed to create a plasma of density $10^{15} \mathrm{~cm}^{-3}$ with $p_{0}=5$ mTorr. All the neutrals entering the plasma at the rate $\Gamma_{i n}$ (equation (9)) are ionized, amounting to $10^{21}$ ionizations $\mathrm{s}^{-1}$ for the parameters of our example. Accounting for inelastic collisions, the average energy expended in creating each ion-electron pair is computed [20] to be about $50 \mathrm{eV}$ at $T_{e}=3 \mathrm{eV}$. Thus a $P_{r f}$ of $8 \mathrm{~kW}$ is required, or only about $16 \mathrm{~kW}$ even if we include a safety factor of two. This extrapolation is highly conjectural and is based on the belief that proper gas handling will overcome the problems encountered in the present experiment. Sakawa et al have achieved densities $>10^{14} \mathrm{~cm}^{-3}$ in a geometry [21] similar to that proposed in figure 13, but the experiment was not pushed to the $10^{15} \mathrm{~cm}^{-3}$ densities envisioned here.

\section{Acknowledgments}

This work was supported by the Semiconductor Research Corporation, the National Science Foundation and the Wisconsin Engineering Research Center for Plasma Aided Manufacturing. We thank David Blackwell, John Evans and Professor Donald Arnush for technical help and discussions.

\section{References}

[1] Boswell R W and Henry D 1985 Appl. Phys. Lett. 471095

[2] Zhu P and Boswell R W 1991 Phys. Fluids B 3869

[3] Chen F F and Chevalier G 1992 J. Vac. Sci. Technol. A 10 1389

[4] Chen F F 1992 Physics of Laser Plasma (Handbook of Plasma Physics 3) ed A M Rubenchik and S Witkowski (Amsterdam: Elsevier) pp 483-517

[5] Chen F F 1990 Phys. Scr. T 3014

[6] Chen F F 1996 Phys. Plasmas 31783

[7] Yasaka Y and Hara Y 1994 Japan. J. Appl. Phys. 335950 
[8] Shinohara S, Miyauchi Y and Kawai Y 1995 Plasma Phys. Control. Fusion 371015

[9] Sakawa Y, Koshikawa N and Shoji T 1996 Appl. Phys. Lett. 691695

[10] Sudit I D and Chen F F 1996 Plasma Sources Sci. Technol. 543

[11] Suzuki K, Nakamura K and Sugai H 1996 Japan. J. Appl. Phys. 354044

[12] Chevalier G and Chen F F 1993 J. Vac. Sci. Technol. A 11 1165

[13] Miljak D G and Chen F F 1998 Plasma Sources Sci. Technol. 761

[14] Sudit I D and Chen F F 1994 Plasma Sources Sci. Technol. 3162
[15] Gilland J, Breun R L and Hershkowitz N 1998 Plasma Sources Sci. Technol. 7416

[16] Light M, Sudit I D, Chen F F and Arnush D 1995 Phys. Plasmas 24094

[17] Blackwell D D 1998 private communication

[18] McDaniel E W and Mason E A 1973 The Mobility and Diffusion of Ions in Gases (New York: Wiley)

[19] Shamrai K P and Taranov V B 1996 Plasma Sources Sci. Technol. 5474

[20] Lieberman M A and Lichtenberg A J 1994 Principles of Plasma Discharges and Materials Processing (New York: Wiley) p 81

[21] Sakawa Y, Koshikawa N and Shoji T 1996 Appl. Phys. Lett. 691695 